\title{
Lattice-Reduction-Aided Receivers for MIMO-OFDM in Spatial Multiplexing Systems
}

\author{
Inaki Berenguer ${ }^{1}$, Jaime Adeane ${ }^{1}$, Ian J. Wassell ${ }^{1}$, and Xiaodong Wang ${ }^{2}$ \\ 1 Laboratory for Communication Engineering \\ Department of Engineering \\ University of Cambridge, Cambridge CB3 0FD39, United Kingdom \\ \{ib226, ja316, ijw24\}@cam.ac.uk \\ 2 Department of Electrical Engineering \\ Columbia University, New York, NY 10027 \\ wangx@ee.columbia.edu
}

\begin{abstract}
Orthogonal Frequency Division Multiplexing (OFDM) significantly reduces receiver complexity in wireless broadband systems and therefore has recently been proposed for use in wireless broadband multi-antenna (MIMO) systems. The performance of maximum likelihood detector in MIMO-OFDM system is optimal, however, its complexity, especially with higher order constellation is prohibitive. A number of other detectors, both linear and non-linear, may offer substantially lower complexity, however, their performance is significantly lower. This paper proposes a class of lattice-reduction-aided (LRA) receivers for MIMOOFDM systems that can achieve near maximum likelihood detector performance with low complexity. Performance comparisons between LRA receiver and other popular receivers, including linear receivers and V-BLAST in both independent and correlated channels, are provided. It will be shown that the performance of LRA receiver is superior as compared to other sub-optimal detection methods, especially when the channel is correlated.

Keywords: lattice reduction, MIMO, OFDM, V-BLAST.
\end{abstract}

\section{INTRODUCTION}

Multiple-input multiple-output (MIMO) technology has recently become very popular since it can improve link reliability without sacrificing bandwidth efficiency. An apparent disadvantage of single-carrier based MIMO systems is the fact that the computational complexity of the receiver (either a vector-MLSE or a multi-channel equalizer) will in general be very high. The use of OFDM alleviates this problem by turning the frequency-selective MIMO channel into a set of parallel narrowband MIMO channels, which will greatly simplify the equalization process. Only a constant matrix has to be inverted for each OFDM tone.

For the detection process, although the performance of maximum likelihood detector is optimal, its complexity is very high. A number of other detectors, both linear and non-linear, offer substantially lower complexity, but their performance is significantly lower. This paper proposes a class of lattice-reduction-aided (LRA) receivers in MIMOOFDM systems that can achieve near maximum likelihood detector performance with low complexity. We extend the LRA receiver technique proposed in [9], applicable for $2 \times 2$ system, to a general $n_{R} \times n_{T}$ system, where $n_{R} \geq n_{T}$. The complexity of LRA receivers is similar to the linear receivers, but its performance is close to that of maximum likelihood (ML) receiver. Performance comparisons between LRA receiver and other popular receivers, includ- ing linear receivers and V-BLAST, will be provided. It will be shown that especially with higher order constellation and when the channel is correlated, LRA significantly outperforms other suboptimal detectors in terms of BER. The remainder of this paper is organized as follows: In Section II, the MIMO-OFDM system model with traditional linear receivers is described. In Section III, the proposed LRA receivers for $n_{R} \times n_{T}, n_{R} \geq n_{T}$ multi-antenna systems are presented. To test the effectiveness of the proposed LRA receivers, simulation results will be provided in Section IV.

\section{BACKGROUND}

\section{A. System Model}

In an OFDM-based spatial multiplexing system the individual data streams are first OFDM-modulated and then transmitted simultaneously from $n_{T}$ antennas. Figure 1 shows an OFDM-based spatial multiplexing system. The modulator applies an $N_{c}$-point IFFT to $N_{c}$ data symbols and prepends the cyclic prefix (CP) of length $L$, which is a copy of the last $L$ samples of the IFFT output. The overall OFDM symbol length is therefore $T=N_{c}+L$. Throughout this paper, we assume $L \leq T / 2$. The CP acts as a guard space between consecutive OFDM symbols and avoids intersymbol-interference (ISI) if the channel impulse response length is less than or equal to the length of the $\mathrm{CP}$. At the receiver, the $\mathrm{CP}$ is removed and an $N_{c}$-point FFT is applied. The signals received by the individual antennas are first passed through OFDM demodulators, separated, demultiplexed, and also potentially decoded.
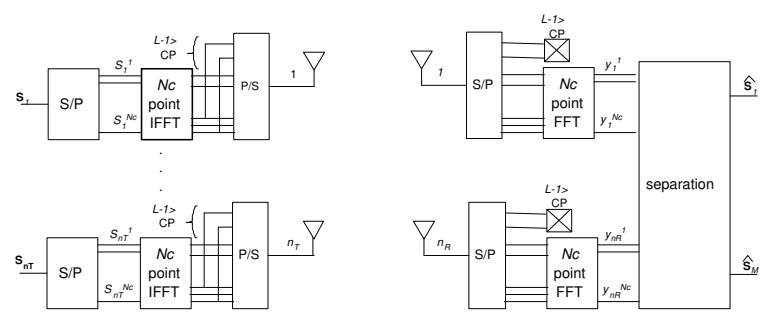

Fig. 1. The block diagram of MIMO-OFDM transceiver.

In Figure 1, the wireless channel for each OFDM carrier can be assumed to be quasi-static and flat, and therefore can be represented by an $n_{R} \times n_{T}$ matrix $\boldsymbol{H}$ whose element 
$h_{i j}$ represents the complex gain of the channel between the $j$-th transmit antenna and the $i$-th receive antenna. The signal received at the $m$-th antenna can now be written as

$$
r_{m}(i)=\sum_{v=1}^{n_{T}} \sum_{l=0}^{L-1} h_{m, v}(l) s_{v}(i-l)+n_{m}(t),
$$

where $n_{m}(t),\left(m=1, \ldots, n_{T}\right)$ is stationary additive (potentially coloured) noise observed at the $m$-th receive antenna during the $i$ th symbol period. Each signal transmitted by the transmit antenna $v$ to receive antenna $m$ is scaled by the $L$-tap multipath channel represented its gains $h_{m, v}$. Using the following notation

$$
\begin{aligned}
\boldsymbol{r}[i] & =\left[r_{1}[i], r_{2}[i], \ldots, r_{n_{R}}[i]\right]^{T}, \\
\boldsymbol{s}[i] & =\left[s_{1}[i], s_{2}[i], \ldots, s_{n_{T}}[i]\right]^{T}, \\
\boldsymbol{n}[i] & =\left[n_{1}[i], n_{2}[i], \ldots, n_{n_{R}}[i]\right]^{T},
\end{aligned}
$$

we can rewrite Eq.(1) in vector-matrix form as

$$
\boldsymbol{r}(i)=\sum_{l=0}^{L-1} \boldsymbol{H}_{l} \boldsymbol{s}(i-l)+\boldsymbol{n}(t)
$$

where $\left[\boldsymbol{H}_{l}\right]_{m, v}=h_{m, v}(l), \quad m=1,2, \ldots, n_{R}, \quad v=$ $1,2, \ldots, n_{T}$ is the $l$-th path between the transmit antenna $v$ and receive antenna $m$. Assuming that the channel is of length $L$ taps, the channel response for the $k$-th carrier can be written as:

$$
\boldsymbol{H}\left(e^{j 2 \pi k / N_{c}}\right)=\frac{1}{\sqrt{N_{c}}} \sum_{l=0}^{L-1} \boldsymbol{H}_{l} e^{-j 2 \pi l k / N_{c}},
$$

for $k=0, \ldots, N_{c}-1$ and $N_{c}$ is the number of OFDM carriers. For notational convenience, in the remainder of this paper we will denote the channel response for the $k$-th carrier, $\boldsymbol{H}\left(e^{j 2 \pi k / N_{c}}\right)$ as $\boldsymbol{H}^{(k)}$. The received signal, transmitted signal, and noise for the $k$-th carrier will be denoted as $\boldsymbol{y}^{(k)}, \boldsymbol{s}^{(k)}$, and $\boldsymbol{n}^{(k)}$, respectively.

Therefore the received signal for the $k$-th carrier can be expressed as:

$$
\boldsymbol{y}^{(k)}=\sqrt{\frac{\rho}{n_{T}}} \boldsymbol{H}^{(k)} \boldsymbol{s}^{(k)}+\boldsymbol{n}^{(k)},
$$

where $\boldsymbol{s}^{(k)}=\left[s_{1}^{(k)}, s_{2}^{(k)}, \ldots, s_{n_{T}}^{(k)}\right]^{T}$ is the transmitted signal vector, $\boldsymbol{n}^{(k)}$ is the $n_{R} \times 1$ received noise vector, and $\rho$ is the signal to noise ratio independent of the transmit antennas. The entries of $\boldsymbol{n}^{(k)}$ are i.i.d. circularly symmetric complex Gaussian variables with unit variance, i.e. $n_{i}^{(k)} \sim \mathcal{N}_{c}(0,1)$.

\section{B. Correlated Channel Model}

The capacity of MIMO systems depend on the spatial properties of the MIMO channel. Partial correlation of channel coefficients affects the capacity of the system. With OFDM structure, for each carrier, the MIMO-OFDM channel can be seen as having multipath flat fading characteristics. It is well known that insufficient antenna spacing

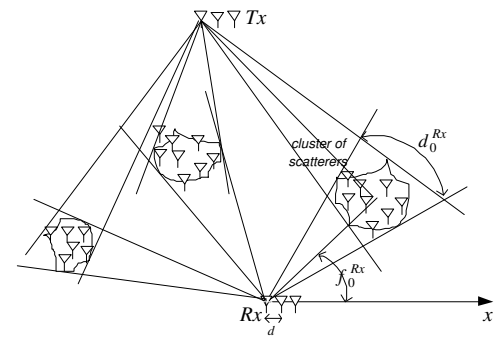

Fig. 2. Geometry of the scattering scenario.

and lack of scattering objects cause the antennas to be correlated. When the channels are correlated at both the transmitter and receiver sides, the channel response for the $l$ th transmission path can be written as [4]

$$
\boldsymbol{H}_{l}=\boldsymbol{A}_{l}^{1 / 2} \boldsymbol{H}_{w} \boldsymbol{B}_{l}^{1 / 2},
$$

where $\boldsymbol{H}_{w}$ is an uncorrelated $n_{R} \times n_{T}$ matrix with i.i.d. $\mathcal{N}_{c}(0,1)$ entries, $\boldsymbol{B}_{l}$ is an $n_{T} \times n_{T}$ transmit covariance matrix for the $l$ th path, and $\boldsymbol{A}_{l}$ is an $n_{R} \times n_{R}$ receive covariance matrix for the $l$ th path. Note that when the $l$ th path is uncorrelated at the transmitter(receiver), $\boldsymbol{B}_{l}=\boldsymbol{I}\left(\boldsymbol{A}_{l}=\boldsymbol{I}\right)$.

Assuming that there is no line of sight between the transmit and receive antennas, the signal at the receive antenna can be seen as originating from a number of clusters or scattering objects. Large values of angular spread $f_{0}^{R x}$ and sufficient antenna separation $d$ result in uncorrelated signals at each of the antennas. In this paper, simulations were conducted in both uncorrelated and correlated channels. In particular, the channel model used is based on modifications of the models in COST-259 [2], [4].

\section{Basic Linear Receivers}

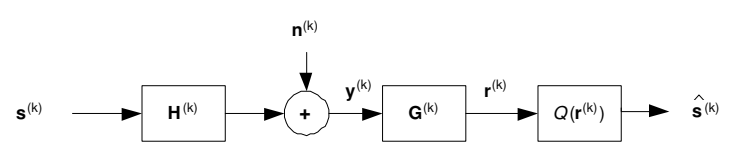

Fig. 3. Traditional linear receiver.

An important feature of linear receivers is their low complexity. With linear receivers, the received signal vector $\boldsymbol{y}^{(k)}$ is linearly transformed by a matrix equalizer $\boldsymbol{G}^{(k)}$ as

$$
\boldsymbol{r}^{(k)}=\boldsymbol{G}^{(k)} \boldsymbol{y}^{(k)}=\sqrt{\frac{\rho}{n_{T}}} \boldsymbol{G}^{(k)} \boldsymbol{H}^{(k)} \boldsymbol{s}^{(k)}+\boldsymbol{G}^{(k)} \boldsymbol{n}^{(k)},
$$

which is later quantized to obtain an estimate of the transmitted symbol vector $\hat{\boldsymbol{s}}^{(k)}=\mathcal{Q}\left(\boldsymbol{r}^{(k)}\right)$. This process is described in Figure 3. For the zero-forcing (ZF) criterion, the equalizer is given by $\boldsymbol{G}^{(k)}=\sqrt{\frac{n_{T}}{\rho}}\left(\boldsymbol{H}^{(k)}\right)^{\dagger}$ where $\left(\boldsymbol{H}^{(k)}\right)^{\dagger}$ denotes the pseudo-inverse of $\boldsymbol{H}^{(k)}$. The $\mathrm{ZF}$ criterion suffers from noise enhancement if at one or several OFDM carriers $k$ the matrices $\boldsymbol{H}^{(k)}$ are rankdeficient or ill-conditioned. On the other hand, the minimum mean-square error (MMSE) linear equalizer, $\boldsymbol{G}^{(k)}=$ 
$\sigma^{2} \sqrt{\frac{\rho}{n_{T}}}\left(\boldsymbol{H}^{(k)}\right)^{H}\left(\frac{\sigma^{2} \rho}{n_{T}}\left(\boldsymbol{H}^{(k)}\right)\left(\boldsymbol{H}^{(k)}\right)^{H}+\sigma_{n}^{2} \boldsymbol{I}_{n_{R}}\right)^{-1} \mathrm{~min}$ imizes the error due to both the noise and interference. There are nonlinear receivers that offer better performance than linear receivers with moderate complexity, such as the BLAST receivers [5], however, in the remainder of this paper we will show that the proposed LRA-receivers are still superior as compared to BLAST.

\section{Lattice-Reduction-Aided Linear ReCEIVERS}

\section{A. Constellation, Lattices, and Basis Change}

Let us consider a real-valued MIMO-OFDM system with $n_{T}=n_{R}=2$, where the transmitted symbols $s_{1}$ and $s_{2}$ belong to a $2 N+1$-PAM constellation, i.e. $s_{i} \in\{-N,-N+$ $1, \ldots, 0, \ldots, N-1, N\}$. Assume that the channel matrix for the $k$-th OFDM carrier is $\boldsymbol{H}^{(k)}=\left[\begin{array}{ll}2 & 3 \\ 1 & 2\end{array}\right]$. Therefore, the received constellation will consist of $\boldsymbol{H}^{(k)} \boldsymbol{s}^{(k)}=$ $s_{1}^{(k)}[2,1]^{T}+s_{2}^{(k)}[3,2]^{T}$. Due to the equalizing operation and the direction of the basis vectors, the decision regions can be seen as parallelograms described by the columns of $\boldsymbol{H}^{(k)}$. In this case, it can be seen that the angle between $\boldsymbol{h}_{1}^{(k)}$ and $\boldsymbol{h}_{2}^{(k)}$ is very narrow and thus a small amount of noise samples can cause the decoder to make a wrong detection.

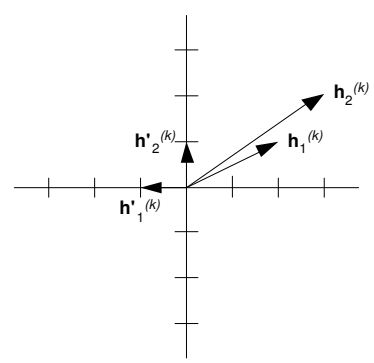

Fig. 4. Original and new bases.

To generalize, the problem with linear receivers is that the decision regions are very narrow when the bases of the lattice are highly correlated. One solution is to find more orthogonal bases for the same lattice to make the decision regions more robust against noise and interference. For example, as can be seen in Figure 4 , the new bases, $\boldsymbol{h}_{1}^{\prime(k)}$ and $\boldsymbol{h}_{2}^{\prime(k)}$ are more orthogonal to each other as compared to $\boldsymbol{h}_{1}^{(k)}$ and $\boldsymbol{h}_{2}^{(k)}$. While a basis change does not always lead to an optimum performance, a change towards more orthogonal bases will, in general, improve the performance. The more correlated the columns of $\boldsymbol{H}^{(k)}$ are, the more significant the improvement in performance is. In this paper, we propose an extension of a reduction technique, firstly appear in [9] for $2 \times 2$ systems, to a general $n_{m} \times n_{t}$ systems.

Theorem [9]: If $\boldsymbol{H}$ is a basis of a lattice, $\boldsymbol{H}^{\prime}=\boldsymbol{H P}$ is also a basis of the same lattice if $\boldsymbol{P}$ and $\boldsymbol{P}^{-1}$ have integer (possibly complex) entries.

For the problem in hand, the objective is to find a change of basis $\boldsymbol{P}^{(k)}$ which transforms the $\boldsymbol{H}^{(k)}$ into $\boldsymbol{H}^{\prime(k)}$, for $k=0, \ldots, N_{c}-1$ such that the decision regions for a specific lattice and decoder are optimized.

\section{B. LRA Receiver}

An input symbol vector of the $k$-th OFDM carrier represented by $\boldsymbol{s}^{(k)}$ in the original basis with elements in $\mathbb{Z}_{\mathbb{C}}$, where $\mathbb{Z}_{\mathbb{C}}$ is set of complex integer, is represented by $\boldsymbol{z}^{(k)}=\left(\boldsymbol{P}^{(k)}\right)^{-1} \boldsymbol{s}^{(k)}$ in the reduced basis. Assuming the received vector $\boldsymbol{y}^{(k)}$ in Eq.(5) is already in the reduced basis,

$$
\boldsymbol{y}^{(k)}=\sqrt{\frac{\rho}{n_{T}}} \boldsymbol{H}^{\prime(k)} \boldsymbol{z}^{(k)}+\boldsymbol{n}^{(k)} .
$$

Since $\boldsymbol{H}^{\prime(k)}=\boldsymbol{H}^{(k)} \boldsymbol{P}^{(k)}$, with the ZF receiver where $\boldsymbol{G}^{(k)}=\sqrt{\frac{n_{T}}{\rho}}\left(\boldsymbol{H}^{(k)} \boldsymbol{P}^{(k)}\right)^{\dagger}$, Eq. (7) can be written as

$$
\boldsymbol{r}^{(k)}=\sqrt{\frac{\rho}{n_{T}}} \boldsymbol{G}^{(k)} \boldsymbol{H}^{(k)} \boldsymbol{P}^{(k)} \boldsymbol{z}^{(k)}+\boldsymbol{G}^{(k)} \boldsymbol{n}^{(k)} \text {. }
$$

The estimate of $\boldsymbol{z}^{(k)}$ is $\hat{\boldsymbol{z}}^{(k)}=\mathcal{Q}\left(\boldsymbol{r}^{(k)}\right)$. Since the lattice points consist of elements in $\mathbb{Z}_{\mathbb{C}}$, the quantization consist of a rounding operation whereby the real and imaginary parts are rounded separately.

To use the lattice theory, the original points in the constellation are required to consist of symbols in $\mathbb{Z}_{\mathbb{C}}$. Note that the origin $[0, \ldots, 0]^{T}$ also belongs to the lattice. Ordinary QAM constellations neither consist of contiguous integers nor contain the origin, and thus, it is necessary to scale and shift the original constellation.

Assuming the shifted and scaled constellation is transmitted, the received signal vector is

$$
\boldsymbol{y}^{\prime(k)}=\sqrt{\frac{\rho}{n_{T}}} \boldsymbol{H}^{(k)} \boldsymbol{s}^{(k)}=\sqrt{\frac{\rho}{n_{T}}} \boldsymbol{H}^{(k)} \frac{1}{2}\left[\boldsymbol{s}^{(k)}+\boldsymbol{d}\right] .
$$

In terms of the signal received when the data bits are transmitted using the original constellation, $\boldsymbol{y}$, Eq.(10) can be rewritten as:

$$
\boldsymbol{y}^{\prime(k)}=\frac{1}{2} \boldsymbol{y}^{(k)}+\frac{1}{2} \sqrt{\frac{\rho}{n_{T}}} \boldsymbol{H}^{(k)} \boldsymbol{d} .
$$

To summarize, the operations at the receiver consist of scaling, shifting, and equalizing in the new basis.

$$
\begin{aligned}
\boldsymbol{r}^{(k)=} & \sqrt{\frac{n_{T}}{\rho}}\left(\boldsymbol{H}^{(k)} \boldsymbol{P}^{(k)}\right)^{\dagger} \frac{1}{2}\left[\sqrt{\frac{\rho}{n_{T}}} \boldsymbol{H}^{(k)} \boldsymbol{P}^{(k)}\left(\left(\boldsymbol{P}^{(k)}\right)^{-1} \boldsymbol{s}^{(k)}\right)\right. \\
& \left.+\boldsymbol{n}^{(k)}+\sqrt{\frac{\rho}{n_{T}}} \boldsymbol{H}^{(k)} \boldsymbol{P}^{(k)}\left(\boldsymbol{P}^{(k)}\right)^{-1} \boldsymbol{d}\right]
\end{aligned}
$$

As a special case, when $n_{T}=n_{R}$ and the channel is of full rank, Equation (12) can be rewritten as

$$
\boldsymbol{r}^{(k)}=\left(\boldsymbol{P}^{(k)}\right)^{-1} \boldsymbol{s}^{(k)}+\frac{1}{2} \sqrt{\frac{n_{T}}{\rho}}\left(\boldsymbol{H}^{(k)} \boldsymbol{P}^{(k)}\right)^{\dagger} \boldsymbol{n}^{(k)} .
$$

The next subsequent steps at the receiver are slicing, returning to the original basis, and undoing the scaling and shifting.

$$
\hat{\boldsymbol{s}}=2 \boldsymbol{P}^{(k)} \mathcal{Q}\left(\boldsymbol{r}^{(k)}\right)-\boldsymbol{d}
$$


Note that the slicing $\mathcal{Q}($.$) is a rounding operation since$ the symbols in the lattice belong to $\mathbb{Z}_{\mathbb{C}}$. In general, LRA receivers are expected have better performance than traditional linear receivers, especially in realistic communication systems scenarios, where the channel is correlated to some degree. In such scenarios, there will be significant gain in performance using LRA linear receivers as compared to traditional linear receivers and V-BLAST.

\section{Basis Reduction Algorithm}

Assume that $\boldsymbol{h}_{1}^{(k)}, \boldsymbol{h}_{2}^{(k)} \in \operatorname{vec}\left(\boldsymbol{H}^{(k)}\right)$ are the bases of the lattice for the $k$-th OFDM carrier. The ideal GramSchmidt orthogonalization will yield Gram-Schmidt coefficient $\mu^{\prime}=\frac{\left\langle\boldsymbol{h}_{1}^{(k)}, \boldsymbol{h}_{2}^{(k)}\right\rangle}{\left\langle\boldsymbol{h}_{2}^{(k)}, \boldsymbol{h}_{2}^{(k)}\right\rangle}$, however, this operation would change the lattice since $\mu^{\prime}$ might not belong to $\mathbb{Z}_{\mathbb{C}}[6]$.

The weakly reduced Gram-Schmidt orthogonalization rounds the real and imaginary parts of the ideal GramSchmidt coefficients separately, i.e. $\mu=\left\lfloor\mu^{\prime}\right\rceil$. This weak reduction method does not change the lattice. An example of basis reduction based on this algorithm is shown in Figure 4.

A notion of lattice reduction for a lattice basis $\boldsymbol{h}_{1}^{(k)}, \ldots, \boldsymbol{h}_{n_{T}}^{(k)}$ was proposed by Lenstra, Lenstra, and Lovasz (LLL) [6]. It uses similar ideas to the Gauss reduction algorithm. For a given $\delta, \frac{1}{4}<\delta<1$, the LLL reduction algorithm modifies an input basis $\boldsymbol{h}_{1}^{(k)}, \ldots, \boldsymbol{h}_{n_{T}}^{(k)}$ such that the resulting basis satisfies the $\delta$-reduction properties

$$
\mu_{i, j} \leq \frac{1}{2} \text { for } 1 \leq j<i \leq n_{T}
$$

and

$$
\delta\left\|\hat{\boldsymbol{h}}_{j}^{(k)}+\mu_{j, j-1} \hat{\boldsymbol{h}}_{j-1}^{(k)}\right\|^{2},
$$

where the vectors $\hat{\boldsymbol{h}}_{1}^{(k)}, \ldots, \hat{\boldsymbol{h}}_{n_{T}}^{(k)}$ denote the Gram-Schmidt orthogonalization of the input basis that can be calculated recursively as

$$
\begin{gathered}
\hat{\boldsymbol{h}}_{1}^{(k)}=\boldsymbol{h}_{1}^{(k)}, \\
\hat{\boldsymbol{h}}_{j}^{(k)}=\boldsymbol{h}_{j}^{(k)}-\sum_{i=1}^{j-1} \mu_{j, i} \hat{\boldsymbol{h}}_{i}^{(k)} \text { for } j=2, \ldots, n_{T},
\end{gathered}
$$

and the Gram-Schmidt coefficients, are equal to

$$
\mu_{j, i}=\frac{\left\langle\hat{\boldsymbol{h}}_{i}^{(k)}, \boldsymbol{h}_{j}^{(k)}\right\rangle}{\left\langle\hat{\boldsymbol{h}}_{i}^{(k)}, \hat{\boldsymbol{h}}_{i}^{(k)}\right\rangle} .
$$

The original LLL algorithm as proposed in [6] can be used to reduce the bases of real vectors. In Algorithm 1, we extend the LLL algorithm in [6] to reduce the bases of general complex vectors.

\section{Algorithm 1: LRA lattice-reduction algorithm}

$$
\begin{aligned}
& \text { Input: Lattice basis } \boldsymbol{h}_{1}^{(u)}=\boldsymbol{H}^{(u)}[:, 1], \ldots, \boldsymbol{h}_{n_{T}}^{(u)}= \\
& \boldsymbol{H}^{(u)}\left[:, n_{T}\right] \in \mathbb{C}^{\ltimes_{\mathbb{T}}} \text {, for } u=0, \ldots, N_{c}-1 \text { and } \frac{1}{4}<\delta< \\
& 1 \quad k=2 \\
& \text { for } u=1, \ldots, N c \text { do } \% \text { for each carrier } \\
& \quad \text { while } k \leq n_{T} \text { do } \\
& \quad \text { for } i=k-1, \ldots, 1 \text { do } \\
& \quad \boldsymbol{h}_{k}=\boldsymbol{h}_{k}-\left[\mu_{k, i} \mid \boldsymbol{h}_{i} ; \%\right. \text { round real and imag } \\
& \quad \text { end for separately } \\
& \quad \text { Compute } \hat{\boldsymbol{h}}_{k} \text { as in }(17) \\
& \quad \text { if } \delta\left\|\hat{\boldsymbol{h}}_{k-1}\right\|^{2}>\left\|\hat{\boldsymbol{h}}_{k}+\mu_{k, k-1} \hat{\boldsymbol{h}}_{k-1}\right\|^{2} \text { then } \\
& \quad \boldsymbol{h}_{k-1} \leftrightarrow \boldsymbol{h}_{k}(\text { exchange) } \\
& \quad k=\max (k-1,2) \\
& \quad \text { else } k=k+1 \\
& \text { end while } \\
& \text { end for } \\
& \text { Output: Reduced lattice basis } \boldsymbol{H}^{\prime(u)}=\left[\boldsymbol{h}_{1}^{(u)}, \ldots, \boldsymbol{h}_{n_{T}}^{(u)}\right] \\
& \text { and } \boldsymbol{P}^{(u)} \text { defined as } \boldsymbol{H}^{\prime(u)}=\boldsymbol{H}^{(u)} \boldsymbol{P}^{(u)}
\end{aligned}
$$

When the rank, which is equal to the number of transmit antenna, $n_{T}=2$ and $\delta=1$, the algorithm above reduces to the Gauss reduction algorithm as proposed in [9]. More orthogonal reduced bases can be obtained by increasing $\delta$ to one, however, this may increase the number of iterations required.

Other basis reduction techniques such as the KorkinKolotarev reduction [3], Minkowski basis [1], Seysen basis [8] and hybrids [7] have different reduction criteria. These bases may have better reduction properties, however, require more computational effort.

\section{Simulation Results}

The simulations presented in this section were conducted with the following parameters: Number of OFDM subcarriers, $N_{c}=16$, number of symbols transmitted per carrier $=1000$, number of transmit antenna $n_{T}=3$, number of receive antenna $n_{R}=3, \delta=1$, and the FIR channel is of length, $L=3$ with 3 resolvable paths. Transmission noise was of complex AWGN $\mathcal{N}(0,1)$. Uncoded system was considered. Gray coding was used for all cases. The multipath channel model used was a modification of SUI-5 model [2]. For correlated channel case, scattering scenario was similar to the ones in COST-259 model. For both the transmitter and receiver side, the mean angle of arrival $(\mathrm{AOA})=45^{\circ}$ and the rms angular spread $=20^{\circ} .50$ independent realizations were simulated for different SNR and the results were averaged.

Remarks:

- Comparing Figure 5- 8, it can be seen that the performance of the LRA receivers approaches the ML receiver as the size of the QAM constellation is increased. This occurs because LRA treats finite constellations as infinite and therefore, data points at the constellation boundary that originally have less neighbours end up having the 


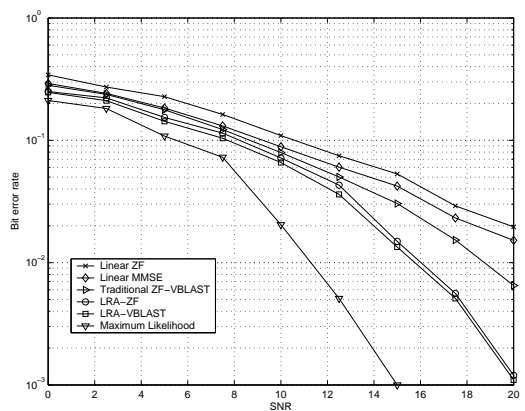

Fig. 5. BER performance of a $3 \times 3$ system with QPSK modulation in uncorrelated channel

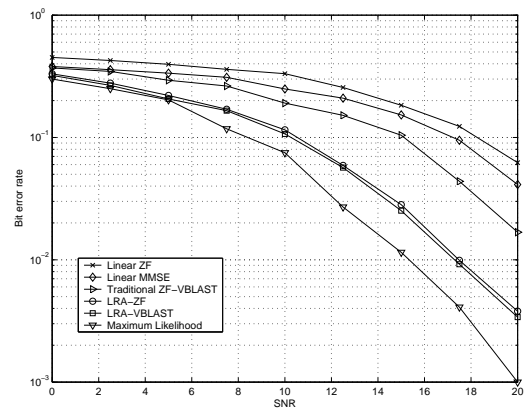

Fig. 6. BER performance of a $3 \times 3$ system with QPSK modulation in correlated channel

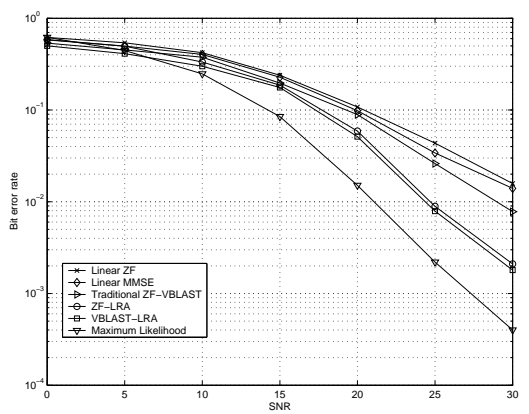

Fig. 7. BER performance of a $3 \times 3$ system with 16 -QAM modulation in uncorrelated channel

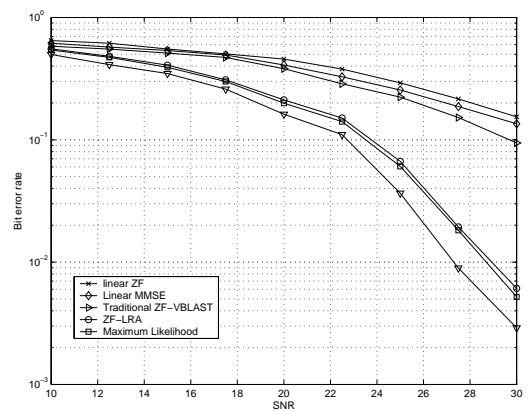

Fig. 8. BER performance of a $3 \times 3$ system with 16 -QAM modulation in correlated channel same number of neighbours as the internal constellation points. This performance loss will be smaller if the ratio between boundary constellation points and internal points is smaller, which is the case with higher order constellations such as 64-QAM and 256-QAM. Since the computational complexity of the ML decoder in high order constellation systems is very high, LRA decoder is a promising alternative, especially if a large number of transmit antennas is used.

- From Figure 6 and 8, it is observed that as the correlation of the channel increases, the performance of the LRA receiver will approach the ML receiver. Also, with high channel correlation, the performance of ZF and MMSE linear receiver are very poor. Since LRA receivers use more orthogonal bases, there are less correlations between the columns of $\boldsymbol{H}^{\prime(k)}, k=0, \ldots, N_{c}-1$, as compared to the original $\boldsymbol{H}^{(k)}$, therefore it performs much better than linear receiver in correlated channel. Therefore, LRA is an alternative method to improve bit error rate performance when the channel correlation is high.

- The two sources of complexity in LRA receivers are in computing the reduced basis of a lattice and to implement the linear equalizer. In quasi-static channels, the lattice is fixed during a block period of data, therefore the basis reduction only has to be performed once for each block period.

\section{Conclusion}

This paper proposes a low complexity receiver structure, based on lattice reduction theory, for $n_{T} \times n_{R}$ MIMO-OFDM system. Simulation results show that the proposed LRA receiver outperforms traditional linear receivers, whereas their complexity are similar. LRA receiver performance approaches ML decoder in higher order constellation system, where the computational complexity of ML decoder is extra orbitant.

\section{REFERENCES}

[1] L. Afflerback and H. Grothe. Calculation of Minkowski-reduced lattice bases. Computing, 35(3-4):269-276, 1985.

[2] B. Asztely. On Antenna Arrays in Mobile Communications Systems: Fast Fading and GSM Base Station Receiver Algorithms. Internal Report S3-SB-9611, KTH, MArch 1996.

[3] A.H. Banihashemi and A. K. Khandani. On the complexity of decoding lattices using the Korkin-Zolotarev reduced basis. IEEE Trans. Inform. Theory, 44(1):162-171, Jan. 1998.

[4] H. Bolcskei, D. Gesbert, A.J. Pauraj. On the capacity of OFDMbased multi-antenna systems. Proc. IEEE Int. Conf. Acoust., Speech, Sig. Proc (ICASSP), Istanbul, Turkey, June 2000.

[5] G.D. Golden, G.J. Foschini, R.A. Valenzuela, and P.W. Wolniansky. Detection algorithm and initial laboratory results using the V-BLAST space-time communication architecture. Electron. Lett., 35(1):14-15, Jan. 1999.

[6] L. Lovasz. An Algorithmic Theory of Numbers, Graphs, and Convexity. Philadelphia, PA: SIAM, 1986.

[7] C. P. Schnorr. A hierarchy of polynomial time lattice basis reduction algorithms. Theor. Comput. Sci., 53(2-3):201-224, 1987.

[8] M. Seysen. Simultaneous reduction of a lattice basis and its reciprocal basis. Combinatorica, 13:363-376, 1993.

[9] H. Yao and G.W. Wornell. Lattice-reduction-aided detectors for MIMO communication systems. In Proc. IEEE Globecom, pages 424-428, Taipei, Taiwan, Nov. 2002. 